\title{
Méthodes d'étude du mode d'action des substan- ces chimiques naturelles ou de synthèse sur le comportement alimentaire des Homoptères
}

\author{
Jacques CHANSIGAUD \& Girard STREBLER (*) \\ I.N.R.A.. Station de Zoologie, Centre de Recherches de Versailles, Route de St-Cyr, F 78000 Versailles \\ (*) I.N.R.A. Paris-Grignon, Laboratoire de Recherches de la Chaire de Zoologie, 16, rue Claude-Bernard, F \\ 75231 Paris Cedex 05
}

RÉSUMÉ

La mise en evidence dans les plantes de substances secondaires jouant un rôle important dans les relations plantes-insectes constitue une nouvelle orientation de recherche, notamment pour la mise au point de méthodes de lutte contre les ennemis des cultures. L'inventaire que nous présentons ici concerne les méthodes utilisées pour l'étude du comportement alimentaire des Homoptères Aphididae et Delphacidae à l'égard de substances végétales primaires ou secondaires.

Mots clés additionnels : Aphididae, Delphacidae, substance allélochimique, stimulus, substrat, relationplante-insecte, preference alimentaire.

Methods for investigating the mode of action of natural or synthetic substances on the feeding behaviour of Homoptera.

The discovery in plants of secondary substances playing an important part in the insect-plant relationship has led to a new orientation of research, particularly in the control of crop pests. This review considers the methods which have been perfected for studying feeding behaviour in Homoptera Aphididae and Delphacidae with reference to primary or secondary substances.

Additional key words : Aphididae, Delphacidae, allelochemical substance, stimulus, substrate, plant-insect relationship. feeding preference.

\section{INTRODUCTION}

Les caractéristiques physiologiques et biochimiques de la plante conditionnent le comportement alimentaire de l'insecte: en effet, un certain nombre de substances chimiques primaires ou secondaires présentes dans la plante inhibent ou stimulent l'alimentation du phytophage. Les substances secondaires ou allélochimiques n'interviendraient pas en général directement dans le métabolisme du végétal, mais elles jouent un rôle important dans les relations planteinsecte.

Les méthodes que nous décrivons dans ce travail peuvent être appliquées à l'étude du mode d'action des substances chimiques naturelles ou de synthèse à l'égard des performances alimentaires des Homoptères, en particulier Aphididae et Delphacidae.
II. LES MILIEUX NUTRITIFS DE SYNTHĖSE : PRÉPARATION, CONSERVATION ET STÉRILISATION DES DIFFÉRENTES SOLUTIONS

Chez les insectes piqueurs comme les Homoptères, le mode d'action des substances chimiques primaires ou secondaires peut être observé notamment après intégration de ces substances dans un milieu aqueux ou un milieu nutritif de synthèse. Lorsqu'il s'agit d'observations d'une durée limitée, les chercheurs emploient soit de l'eau soit une solution aqueuse de saccharose. Par contre, pour des essais qui se prolongent sur une période relativement longue, il existe des solutions nutritives dont la composition chimique est à même de couvrir les besoins de croissance et de 
développement de l'aphide pendant une ou plusieurs générations consécutives.

Les premières tentatives d'élevage d'aphides sur milieu de synthèse sont dues à HAMILTON (1935). Toutefois ce n'est que beaucoup plus tard que des expériences entreprises avec Myzus persicae Sulz. (MITTLER \& DADD, 1962) mais aussi avec d'autres pucerons comme Acyrthosiphon pisum Harr. (AUClair \& CARTIER, 1963), Aphis fabae Scop., Neomyzus circumflexus Buckt (DADD \& KRIEGER, $1967 a$; EHRHARDT, 1968) ont été couronnées de succès. Par contre, les tentatives d'élevage des pucerons des céréales sur milieux nutritifs de synthèse se sont avérées décevantes jusqu'à ce jour.

La composition chimique de la solution nutritive est basée sur celle de la sève élaborée de la plante. Divers auteurs ont monté qu'il y avait environ une vingtaine d'acides aminés indispensables à la survie de $M$. persicae (tabl. 1).

Parmi les facteurs qui conditionnent la réussite de l'alimentation artificielle de l'insecte, la pureté du solvant utilisé dans la préparation du milieu joue un rôle très important : il faut utiliser de l'eau qui, dans un premier temps, doit être désionisée puis distillée dans un appareil en verre.

Certaines substances en solution aqueuse précipitent et, de ce fait, il est indispensable de respecter un ordre de dissolution: le saccharose est en général la $1^{\text {re }}$ substance dissoute dans l'eau ; il est suivi des acides aminés, des sels et des vitamines. La dissolution des sels nécessite une agitation afin de faciliter leur intégration dans la solution, d'autant plus que certains d'entre eux sont utilisés à la limite de leur solubilité en solution aqueuse.

Les acides aminés, les vitamines et les sels en solution doivent être conservés séparément au congélateur à une température de $-20^{\circ} \mathrm{C}$. Les diverses solutions sont par la suite décongelées, en fonction des besoins, puis diluées et mélangées au saccharose.

Le milieu complet peut être stocké au congélateur.
Dans ce dernier cas, il est conseillé de fractionner au préalable la solution nutritive de manière à disposer du volume de solution nécessaire à la confection des sachets que l'on envisage d'utiliser ; cela évite la détérioration du milieu consécutive aux transferts répétés de celui-ci du froid à la température ambiante.

Au moment de l'emploi de la solution nutritive, il est indispensable de stériliser celle-ci dans une enceinte métallique avec filtre et préfiltre afin d'éviter le développement trop rapide des microorganismes. Ce matériel et l'aiguille qui sert à déposer le liquide sur la membrane de parafilm doivent au préalable être désinfectés par passage à l'autoclave à $120^{\circ} \mathrm{C}$ pendant $20 \mathrm{mn}$. La membrane de Parafilm est traitée également par exposition à des rayons U.V. pendant $20 \mathrm{mn}$.

Le pH du milieu peut avoir une incidence non négligeable sur la physiologie de l'aphide : AucLaIR (1965) note une réduction de la croissance, de la reproduction et de la survie de l'aphide du pois en relation avec la valeur du $\mathrm{pH}$.

\section{DISPOSITIF PERMETTANT L'ÉTUDE DU COMPORTEMENT ALIMENTAIRE DES HOMOPTĖRES SUR MILIEUX ARTIFICIELS OU SUR LE VÉGÉTAL}

Il existe dans la littérature un certain nombre de descriptions relatives à la réalisation d'enceintes spécialement conçues pour l'élevage des pucerons et des cicadelles sur milieux artificiels. Certains de ces dispositifs, notamment ceux de MitTler \& DADD (1964a) sont couramment utilisés par les chercheurs.

Les différents types d'enceintes permettent de proposer à l'insecte une ou plusieurs sources alimentaires pour l'étude, selon le cas, de l'effet isolé ou simultané des substances secondaires intégrées dans une seule ou plusieurs solutions nutritives.

TABLEAU 1

Composition du régime artificiel pour M. persicae du DADD-MITTLER, 1966 (quantité de produits pour 100 ml d'eau). Composition of artificial diet for Myzus persicae (quantities per $100 \mathrm{ml}$ water).

L. Acides aminés (*) en mg

Alanine

Arginine

Asparagine

Acide aspartique

Hydrochlorure de cystéine

Acide glutamique

Glutamine

Glycine

Histidine

Isoleucine

Leucine

Hydrochlorure de lysine

Méthionine

Phénylalanine

Proline

Sérine

Thréonine

Tryptophane

Tyrosine

Valine

100
270
550
140
40
140
150
80
80
80
80
120
40
40
80
80
140
80
40
80

Saccharose (en g)

15,0

Vitamines, sels etc... en mg

Acide ascorbique

Thiamine

Riboflavine

Acide nicotinique

Pyridoxine

Acide folique

Pantothénate de $\mathrm{Ca}$ Inositol

Chlorure de choline

Biotine $\mathrm{PO}_{4} \mathrm{KH}_{2}$

$\mathrm{Mg} \mathrm{Cl} \mathrm{Cl}_{2} 6 \mathrm{H}_{2} \mathrm{O}$

$\mathrm{Fe}$ (sous forme de séquestrène)

$\mathrm{Zn}$ (sous forme de séquestrène)

Mn (sous forme de séquestrène)

$\mathrm{Cu}$ (sous forme de séquestrène)

(*) La pureté des isomères est sujette à critique. 


\section{A. Contrôle de l'activité d'une substance secondaire}

AUCLAIR (1965) dans une étude sur la nutrition concernant l'aphide du pois a mis au point le dispositif suivant : $0,3 \mathrm{ml}$ du régime alimentaire sont déposés dans une poche constituée de 2 membranes de Parafilm non étirées : la $1^{\text {re }}$ membrane constitue la partie incurvée de la poche à l'intérieur de laquelle est placé le liquide ; ce dernier est ensuite recouvert par une $2^{\mathrm{e}}$ membrane. La poche ainsi formée est fixée sous un tube de verre (de $25 \mathrm{~mm}$ de long et $18 \mathrm{~mm}$ de diamètre) autour duquel est disposé un anneau en caoutchouc. La partie inférieure du dispositif comporte une petite bouteille en verre munie d'une rainure et d'une arête circulaire autour de l'ouverture, ce qui permet d'ajuster l'anneau de caoutchouc. L'enceinte est ventilée au moyen de 2 orifices de 3 à $4 \mathrm{~mm}$ de diamètre pratiqués sur les côtés, selon le procédé décrit par LAFRANCE (1963). L'humidité de l'enceinte est maintenue à l'aide d'un tampon de coton imprégné d'eau et déposé au fond de la bouteille (fig. 1).

Le dispositif mis au point par MITTLER \& DADD (1964a) est constitué d'un anneau en matière plastique (de $25 \mathrm{~mm}$ de long et de 20 à $25 \mathrm{~mm}$ de diamètre), fermé à la partie supérieure par un sachet à contenu nutritif et à la partie inférieure par un bouchon de liège. Le sachet est composé de 2 membranes de Parafilm étirées adhérant à la paroi de l'anneau : la $1^{\text {re }}$ sert de support à la solution nutritive et la $2^{\mathrm{e}}$ recouvre le liquide. Le volume de la goutte de solution et la pression exercée par la $2^{\mathrm{e}}$ membrane déterminent la surface occupée par le liquide qui peut ainsi s'étendre sur toute la surface réservée à l'alimentation de l'aphide. Cependant, afin de favoriser le scellement des 2 membranes autour de la goutte, il est conseillé d'éviter que la solution déborde sur les parois de l'anneau. Une fois enfermés dans l'anneau clos par le bouchon de liège, les pucerons s'alimentent en insérant leurs

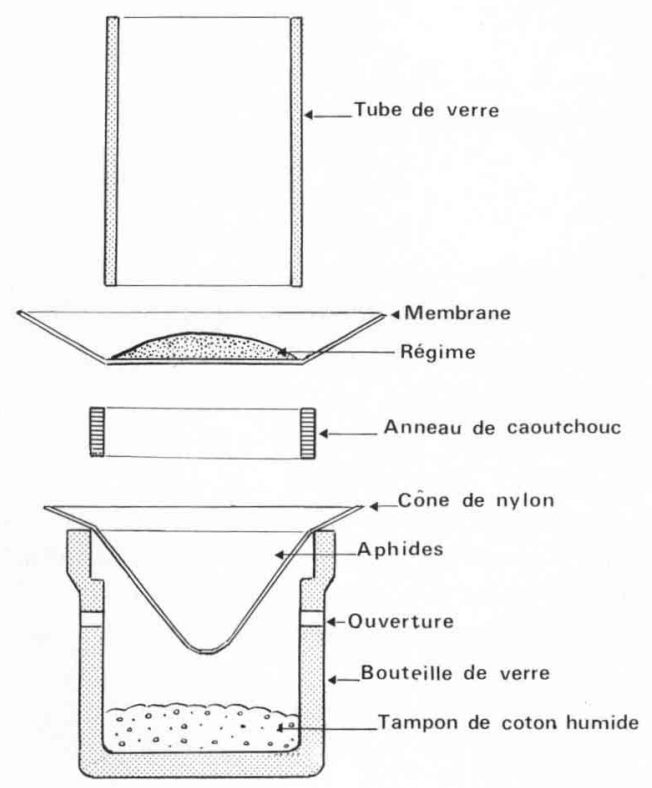

Figure 1

Diagramme des parties qui composent l'appareil d'alimentation de l'aphide.

Diagram of parts that constitute the apparatus for aphid feeding (A৫Cl.tIR, 1965) stylets dans la membrane inférieure de Parafilm (fig. 2).

YOSHIHARA et al. (1979) ont mis au point une cage dans le but d'étudier le comportement alimentaire de Nilaparvata lugens Stål, à l'égard de l'acide silicique. La chambre d'alimentation comprend une cloche en matière plastique (de $2,5 \mathrm{~cm}$ de haut) reposant sur un verre de montre (de $7,5 \mathrm{~cm}$ de diamètre) à l'intérieur duquel sont déposés $5 \mathrm{ml}$ de solution nutritive recouverts par une membrane étirée de Parafilm (fig. 3). L'ensemble du matériel est ensuite placé à l'intérieur d'une chambre obscure sur un support comprenant un certain nombre d'orifices (de $5 \mathrm{~mm}$ de diamètre) permettant le passage de rayons lumineux émis par des tubes fluorescents. La lumière émise est filtrée par du papier cellophane jaune afin d'attirer les cicadelles qui se concentrent ainsi sur la membrane de Parafilm et prélèvent la solution à étudier. Notons que ce dispositif de filtration de la lumière est également préconisé pour l'étude du comportement alimentaire de $M$. persicae (fig. 3).

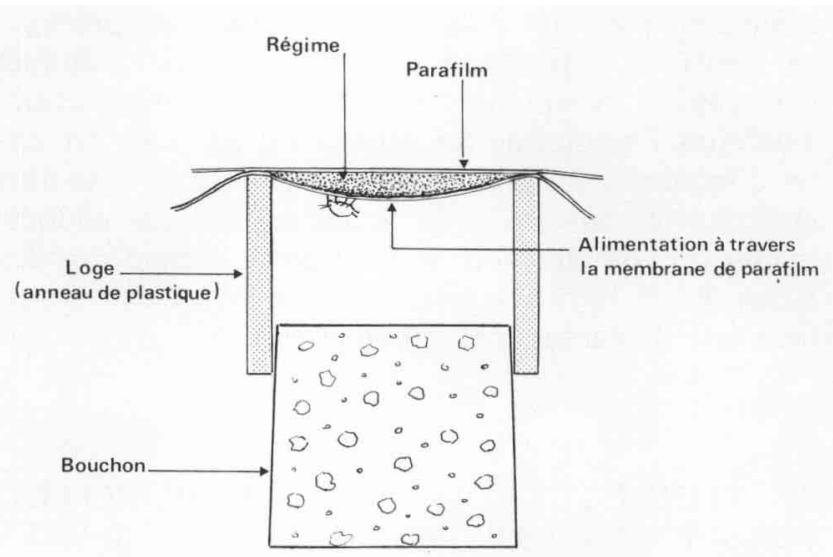

Figure 2

Elevage des aphides sur régimes artificiels.

Rearing aphids on artificial diets (MITTIL:R \& DADI), 1964a)

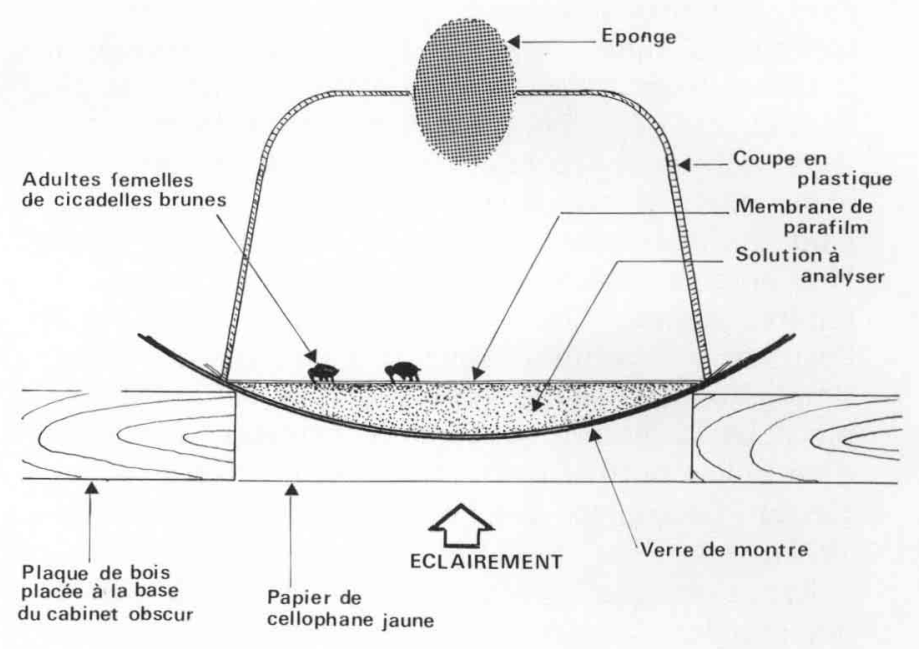

Figure 3

Appareil utilisé pour l'étude du comportement alimemaire de Nilaparvata lugens Stäl. sur des extruits de riz.

Apparatus used for the study of Nilaparvata lugens Stäl. feeding behaviour on rice extracts (YOSHIHARA, 1979). 


\section{B. Contrôle de l'activité de plusieurs substances secondaires par un procédé de choix}

CARTIER \& Auclair (1964), Honeyborne (1969) ont mis au point un dispositif de choix. Il s'agit d'une boîte de Petri dont le couvercle est percé de plusieurs orifices, dans lesquels sont insérés des tubes dont la partie inférieure est fermée par des sachets en Parafilm qui renferment différents régimes ou milieux colorés dans le but d'étudier le comportement de sélection de l'aphide (fig. 4).

\section{Contrôle effectué sur le végétal}

Les expérimentations effectuées sur le végétal sont entreprises fréquemment avec des cages munies de pinces. La constitution et les dimensions de semblables cages varient d'une expérience à l'autre. Le principe du dispositif de la cage est le suivant : 2 anneaux de Plexiglas sont maintenus en regard l'un de l'autre, de part et d'autre du limbe de la feuille, à l'aide d'une petite pince. Le bord des anneaux est garni avec de la mousse de plastique polystyrène de manière à protéger les tissus de la feuille. L'anneau à l'intérieur duquel sont placés les aphides est recouvert de mousseline. Toutefois l'emploi de ces cages n'est pas sans présenter d'inconvénients : l'intérieur de la cage est assombri et la partie couverte de la feuille est souvent endommagée provoquant ainsi la sénescence prématurée des tissus. C'est la raison pour laquelle les enceintes doivent être déplacées fréquemment (fig. 5).

\section{TECHNIQUES DE CONTRÔLE DE L'EFFET DES SUBSTANCES PRIMAIRES OU SECONDAIRES PRÉSENTES DANS DIFFÉRENTS SUBSTRATS ALIMENTAIRES SUR LA PRISE DE NOURRITURE}

La plupart des méthodes de contrôle peuvent être appliquées à des expérimentations effectuées soit sur le végétal, soit sur un milieu nutritif artificiel. Cependant, certaines de ces méthodes ne peuvent être utilisées sur un milieu de synthèse que dans la mesure où le régime alimentaire assure le développement complet de l'insecte. Les contrôles concernant l'application d'une substance secondaire en solution sont généralement entrepris sur de courtes périodes, souvent pendant quelques heures seulement, dès l'instant oủ les Homoptères consentent à effectuer des sondages ou piqûres d'essai. Dans ces conditions, on utilise de l'eau ou une solution aqueuse de saccharose ; cette simplicité du milieu de base peut s'avérer indispensable dans la mesure où certains éléments constitutifs d'un milieu nutritif peuvent exercer un effet de synergie sur la substance que l'on veut étudier (KLINGAUF, 1971).

Rappelons que l'étude de la stimulation alimentaire des aphides par des constituants des régimes artificiels, dans lesquels sont éventuellement incluses des substances secondaires, peut être effectuée de 2 façons:

a) Par des analyses de choix (MitTler \& Dadd, $1964 b$ ) : les aphides ont alors accès à 2 ou plusieurs

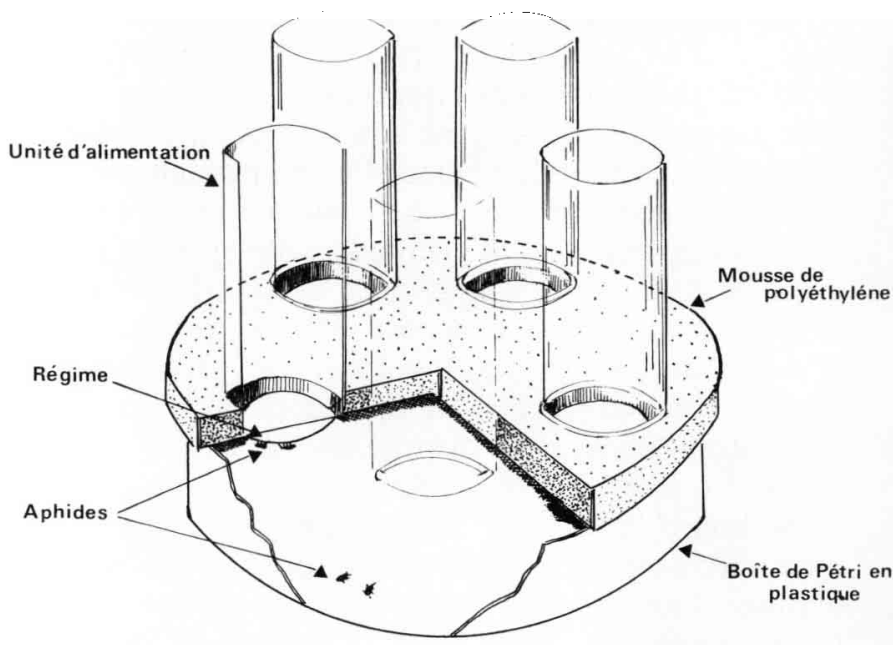

Figure 4

Chambre dans laquelle les aphides peuvent choisir entre des régimes artificiels contenant diverses substances de croissance ou des substances secondaires.

Chamber in which aphids can select between artificial diets containing various growth substances or secondary substances HONEYBORNE, 1969).

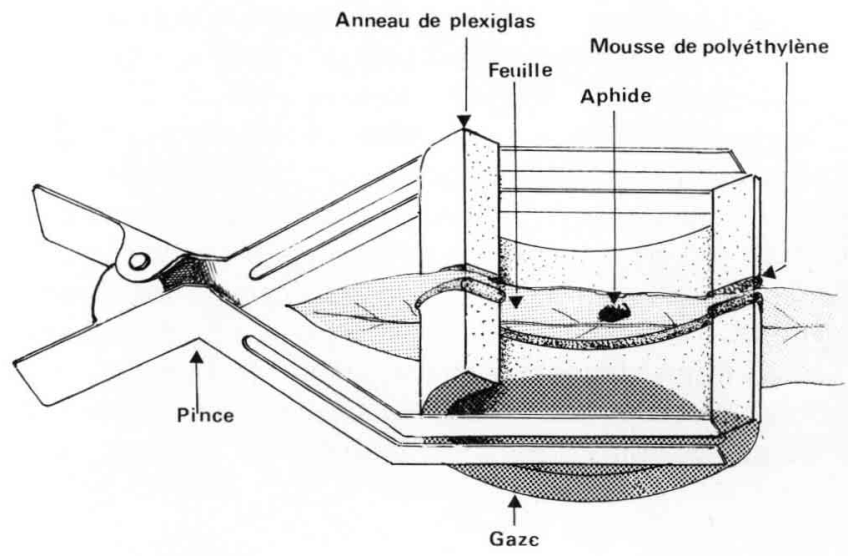

Figure 5

Structure d'une cage à pince.

Structure of a clip-on cage (HONEYBORNE, 1969).

substances différentes, la préférence diététique étant estimée par un comptage périodique des individus installés sur chacun d'entre eux.

b) Par l'estimation de la proportion d'aliment ingéré ; cette autre mesure de la phagostimulation est utilisée principalement dans des situations de nonchoix, après adjonction de colorants ou de radiotraceurs aux solutions nutritives ou simplement par le contrôle du poids des sachets à contenu nutritif avant et après l'alimentation (AUCLAIR, 1965 ; MITTLER, 1967).

\section{A. Technique de pesage}

A partir d'un milieu de synthèse, DADD \& KRIEGER $(1967 b)$ se réfèrent au poids de l'aphide dans le but de mesurer la performance de celui-ci. Ces auteurs déterminent le poids de $M$. persicae $8 \mathrm{j}$ après sa naissance, 
à partir du moment où les individus du lot témoin atteignent leur complet développement. Toutefois ce procédé ne peut être appliqué aux substances secondaires que dans la mesure où l'expérimentateur dispose d'un milieu nutritif qui assure l'évolution de l'insecte pendant plusieurs générations consécutives.

DADD \& MitTler (1965) puis EHRHARDT (1968) pèsent à 2 ou $3 \mathrm{j}$ d'intervalle les aphides qui s'alimentent sur différents régimes et comparent les courbes de croissance. Au cours d'un travail similaire, MiTTLER \& DADD (1964c) emploient une mesure d'accroissement amplifiée, soit l'accroissement total de poids journalier de toutes les larves, soit le poids cumulé du matériel aphide durant le temps de l'expérimentation. AUClair (1965), MitTler (1970) évaluent, quant à eux, la quantité de nourriture ingérée par la perte de poids des sachets à contenu nutritif au cours de la période d'alimentation. MASSONIE (1980) apprécie l'ingestion à partir du rapport du poids total de nourriture ingérée sur le poids initial de l'ensemble des pucerons.

\section{B. Durée des piqûres d'essai}

Parmi les différents paramètres couramment utilisés, il faut noter la durée des piqûres d'essai consécutive à l'insertion des stylets dans le milieu nutritif. Sur le milieu nutritif à tester, la membrane supérieure de Parafilm de la cagette de MITTLER recouvrant normalement le liquide est remplacée par une lamelle de verre qui permet des observations relatives aux mouvements des stylets de l'aphide au cours des piqûres d'essai.

La pénétration des stylets dans la solution ou dans le tissu végétal suivie de l'immobilisation du puceron correspond à une période d'alimentation. Sa durée est déterminée sous la loupe binoculaire au moyen d'un chronomètre. MONTGOMERY \& ARN (1974) ont enregistré la durée des piqûres d'essai d'Aphis pomi De Geer, de $M$. persicae et d'Amphorophora agathonica Hot. à partir du moment où le rostre de l'aphide, placé à angle droit au contact de la membrane, reste immobile pendant au moins $5 \mathrm{~s}$.

\section{Longévité}

La longévité des pucerons adultes sur un milieu de synthèse ou sur une partie de la plante constitue un élément d'appréciation du comportement alimentaire. Dans ce contexte, Auclair (1965), MitTler \& DADD (1963b) VAN EMDEN (1967), WEARING \& VAN EMDEN (1967) se servent d'une échelle «log-probit», exactement comme s'il s'agissait d'essais insecticides; la mortalité est exprimée en terme de probit et le temps d'exposition au régime alimentaire $(J+1)$ en logarithme. Une valeur représentant le logarithme du temps pour une mortalité de 50 p. 100 est ensuite déterminée pour chaque groupe d'aphides (fig. 6).

\section{Fécondité}

La fécondité de l'aphide peut être prise en compte en vue de contrôle de la valeur de l'aliment, avec

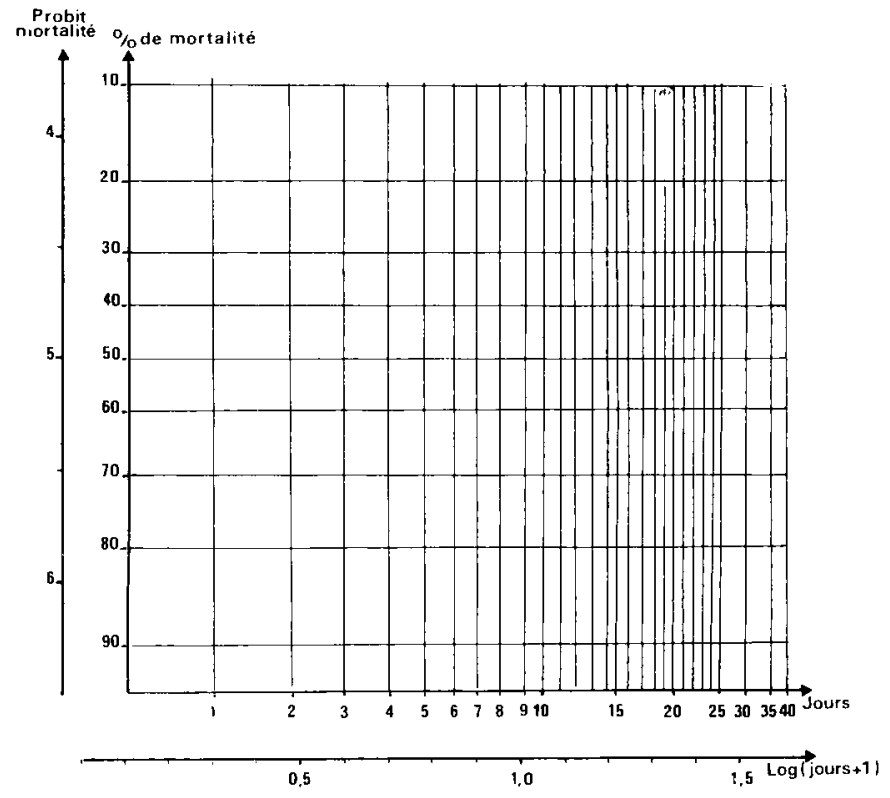

Figure 6

Papier graphique réalisé pour le tracé direct de survie des aphides. Graph developed for the direct plotting of aphid survival (MITTLER \& DADD, 1963b).

cependant les réserves qui s'imposent dans le cas d'un milieu nutritif artificiel pour les raisons que nous indiquions précédemment. Sur le végétal, certains chercheurs considèrent que ce critère de contrôle est supérieur à celui qui a trait à la détermination des temps d'installation de l'homoptère sur le substrat alimentaire (NAult \& STYER, 1972). MASSONIE (1980), au cours d'une étude concernant l'influence de l'acide oxalique et de l'acide gentisique sur la valeur nutritive pour $M$. persicae d'un milieu synthétique, évalue tout d'abord celle-ci en se basant sur la survie des pucerons, puis en seconde approche sur la fécondité de la $3^{\mathrm{e}}$ génération.

\section{E. Coloration de la solution}

La coloration de la solution, que celle-ci soit une solution nutritive simple ou plus complète, comprenant une substance secondaire, constitue un élément d'appréciation du comportement alimentaire de l'homoptère. Tous les colorants sont plus ou moins nocifs : le rouge neutre à 0,1 p. 100 ne peut être utilisé que pour des observations de courte durée (MITTLER \& DADD, 1963a). La solution colorée est perçue à l'intérieur du tube digestif du puceron. Une échelle de coloration peut être établie afin d'évaluer les quantités relatives de solution ingérée. Cette évaluation est basée sur l'intensité et l'étendue de la coloration de l'œsophage de l'aphide observé à travers la cuticule dorsale. Lorsqu'il s'agit d'un cas douteux ou d'un adulte ailé, l'aphide est disséqué. MitTler \& DADD $(1963 a)$ ont établi un système de notation allant de 0 à 3 :

$0=$ Individus pour lesquels aucune coloration n'est observée ou définissable.

$1 / 2=$ Individus dont l'œsophage est coloré en rouge, (et/ou) avec une coloration rose à peine perceptible à la partie antérieure du jabot. 
1 = Individus chez lesquels, en plus de la coloration de l'œsophage, la partie antérieure du jabot est colorée en rouge. Cette notation s'applique également aux pucerons pour lesquels l'ensemble du jabot est profilé en rouge ou d'une couleur légèrement rose.

$2=$ Individus chez lesquels le jabot est entièrement coloré en rouge en l'absence de couleur dans le milieu intestinal et au-delà du jabot.

$3=$ Individus chez lesquels la coloration rouge s'étend à l'ensemble de l'intestin. Dans cette catégorie sont inclus les pucerons qui présentent une coloration diffuse de l'extrémité de l'intestin au niveau de la région anale.

La prise de nourriture est estimée de la façon suivante :

a) d'après le pourcentage d'individus qui ne présentent aucune coloration;

b) d'après le nombre de pucerons appartenant à chaque gradient de coloration rapporté à la totalité des individus de chaque traitement ;

c) d'après le nombre de colorations observées, nombre découlant de la somme des valeurs obtenues en multipliant chaque gradient de coloration par la fréquence des individus appartenant à celui-ci.

\section{F. Taille et fréquence des fourreaux salivaires}

Le nombre plus ou moins élevé de fourreaux salivaires donne des informations sur la fréquence des prises alimentaires des pucerons sur des milieux comprenant différentes substances chimiques primaires ou secondaires.

Les sécrétions salivaires interviennent au moment où les aphides insèrent leurs stylets dans la membrane de Parafilm. Deux formes caractéristiques de sécrétions sont observées :

a) Des fourreaux salivaires simples ou ramifiés, comparables à ceux qui se forment lorsque l'insecte enfonce ses stylets dans les tissus de la plante ou à l'intérieur de membranes (NICKEL \& SYLVESTER, 1959).

b) De petites accumulations salivaires qui apparais sent sur la membrane à la suite d'une insertion partielle des stylets ; ces «salivations ou pâtés » sont ainsi dénommés parce qu'ils ne représentent pas de tubes salivaires de manière évidente.

L'identification des fourreaux salivaires après leur dépôt sur la membrane de Parafilm est effectuée après coloration de ceux-ci au rouge neutre ou à la safranine.

MiTTLER \& DADD (1965) effectuent le dénombrement des fourreaux salivaires par observation au microscope. Chaque membrane colorée est placée audessus d'une surface de papier millimétré ; les fourreaux salivaires et les pâtés présents à l'intérieur de 25 surfaces unitaires de $1 \mathrm{~mm}^{2}$ sont comptés.

Au cours d'une étude du comportement alimentaire d'Homoptères Delphacidae-Deltocephalidae, SEKIDO \& SogAWA (1976) ont coloré les fourreaux salivaires émis par les cicadelles avec une solution aqueuse à 0,1 p. 100 de rhodamine B. La longueur moyenne et les proportions de fourreaux allongés ou ramifiés sont prises en considération pour l'évaluation des réponses.
NAult \& STyer (1972) colorent les émissions salivaires d'aphides placés sur des solutions contenant de la sinigrine avec de la safranine. Les fourreaux salivaires émis par les pucerons qui s'alimentent à partir d'une solution aqueuse de sinigrine atteignent une longueur plus importante que chez les pucerons alimentés avec de l'eau ( $12 \mu \mathrm{m}$ contre $2,3 \mu \mathrm{m})$.

L'action inhibitrice des amines aromatiques sur la prise de nourriture d'Homoptères DelphacidaeDeltocephalidae est mesurée colorimétriquement par KURATA \& SOGAWA (1976) en ajoutant aux solutions du naphtol jaune $S$ (2,4 dinotro 1 naphtol-7-8) acide sulfonique) à la concentration de 0,02 p. 100 .

Le coefficient d'adsorbance des solutions collectées est mesuré à $430 \mathrm{~nm}$ avec un spectrophotomètre. La valeur d'imbibition est calculée par la formule suivante :

$$
x=y(y+z) \frac{A-B}{A(y+z)-\bar{B} y}
$$

où $x$ est égal à la quantité de liquide imprégnant l'insecte,

y est égal à la quantité de solution enfermée dans le sachet de parafilm,

z est égal à la quantité d'eau distillée nécessaire à la dilution,

A est égal au coefficient d'absorbance avant la prise de nourriture de l'insecte,

B est égal au coefficient d'absorbance après la prise de nourriture de l'insecte.

Le degré d'imprégnation est calculé par rapport à une courbe d'étalonnage.

\section{G. Collecte de miellat}

Ce procédé est surtout utilisé pour des études sur la plante. Il constitue une source d'information non négligeable quant au degré d'appétence d'une plantehôte à l'égard de l'aphide. Une substance secondaire pulvérisée à la surface du végétal, circulant ou non à l'intérieur de la plante ou d'un fragment de celle-ci, peut également être testée de cette façon.

BANKS \& MACAULAY (1964), au cours d'une expérimentation de longue durée avec $A$. fabae sur le végétal, font une estimation de la quantité de miellat excrété par récupération de celui-ci dans de l'huile. Une boîte de $2,3 \mathrm{~cm}$ de diamètre contenant de l'huile est disposée sous chaque aphide. Les enregistrements du nombre de gouttelettes par aphide $(\bar{f})$ sont groupés en périodes appropriées à partir du moment où les volumes moyens $\bar{v}$ des gouttelettes sont estimés. La moyenne des volumes de miellat excrétés est le produit $\overline{\mathrm{f}} \cdot \overline{\mathrm{v}}$ dont la variance est $\left[\overline{\mathrm{v}}^{2} \cdot \mathrm{s}^{2}(\overline{\mathrm{f}})+\overline{\mathrm{f}}^{2} \cdot \mathrm{S}^{2}(\overline{\mathrm{v}})\right]$ ou $S^{2}(\bar{f})$ et $S^{2}(\bar{v})$ sont respectivement la variance de la fréquence moyenne d'excrétion et du volume moyen de la gouttelette. Les auteurs convertissent le volume en poids de miellat par détermination de la gravité spécifique de celui-ci à partir de collectes opérées à l'aide de tubes capillaires en verre de précision d'une capacité de $2 \mathrm{~mm}^{3}$.

L'huile minérale employée pour la récolte de miellat, du type S.A.E. 30, de densité 0,89 est de couleur brun clair ; les gouttelettes de miellat transparentes, 
de forme sphérique, restent en suspension dans le liquide juste au-dessous de la surface de l'huile. L'expérimentateur peut alors mesurer le diamètre des gouttelettes avec un micromètre oculaire et en déduire le volume moyen $(v)$.

Au cours d'une expérimentation effectuée sur le végétal, HONEYBORNE (1969) collecte le miellat dans une boîte de Pétri à l'intérieur de laquelle il a disposé de l'huile dont la gravité spécifique est de l'ordre de 0,7 (VAN EMDEN, 1972). La boîte est placée à une faible distance des pucerons afin de limiter les pertes par évaporation au cours de la chute des gouttelettes. Le diamètre de la boîte est choisi après évaluation de la dispersion des gouttelettes de miellat sur une feuille de papier buvard imprégné avec du bleu de bromocrésol ( $2 \mathrm{mg} / \mathrm{ml}$ d'alcool éthylique) ; ce papier passe de la couleur jaune à la couleur bleue au contact du miellat. L'auteur conseille d'enduire les parois de la boîte avec un silicone afin d'empêcher le miellat d'adhérer au verre de l'enceinte. Avant l'emploi, il est ajouté à l'huile une solution de saccharose à $10 \mathrm{p}$. 100 et le mélange est agité puis laissé en suspens ce qui évite un échange important d'eau entre les gouttelettes de miellat et l'huile. A la fin de la période de collecte, l'huile contenant les gouttelettes de miellat est centrifugée dans un tube capillaire de $1 \mathrm{~mm}$ de diamètre. La quantité de miellat collectée peut être mesurée d'après la longueur de capillaire occupée par celui-ci.

KIM et al. (1975) analysent la résistance d'une graminée Echinochloa crusgalli P.B. à l'égard de la cicadelle Nilaparvata lugens Stål en fonction de la quantité de miellat excrétée. De façon analogue au procédé de Sogawa \& PATKAK (1970), 10 femelles adultes sont introduites dans une cage en plastique où elles s'alimentent pendant $24 \mathrm{~h}$. Recueillie sur un papier filtre, la quantité de miellat excrétée par les insectes est estimée d'après l'intensité de la coloration du papier à la suite d'un traitement à la ninhydrine (fig. 7).

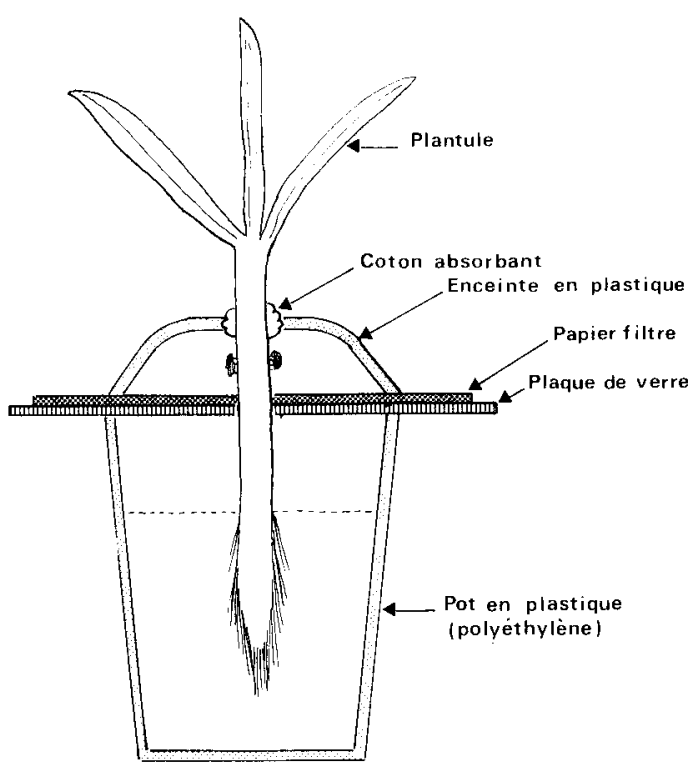

Figure 7

Appareil pour collecter le miellat.

Apparatus for collecting honeydew (SOGAWA, 1970).

\section{H. Les radioisotopes}

La facilité de détection des isotopes radioactifs à de faibles niveaux d'activité a entraîné leur emploi dans les recherches entomologiques. La manipulation de ces substances n'est toutefois pas sans danger et les radiations peuvent avoir des effets létaux et sublétaux sur les insectes marqués.

Les isotopes qui émettent un rayonnement $\gamma$ ont l'avantage d'être détectés à des concentrations plus faibles que les isotopes à rayonnement $\alpha$ ou $\beta$; leur emploi est donc particulièrement justifié dans le cas d'une évaluation quantitative. Cette évaluation est faite par écrasement du matériel sur une planchette métallique, laquelle est ensuite examinée à l'aide d'un tube Geiger couplé à un compteur de scintillation.

La persistance d'un isotope, laps de temps au cours duquel celui-ci reste détectable, est caractérisée par sa demi-vie (indiquée dans les tables de Hollander et al., 1953). Au cours des expérimentations, la demi-vie effective de la substance radioactive est vraisemblablement un peu plus courte que celle qui est indiquée dans les tables, des atomes de l'isotope passant dans les tissus. Ainsi, la demi-vie du ${ }^{32} \mathrm{P}$ est réduite de 14 à $7 \mathrm{j}$ lorsque l'isotope est ingéré par Anthonomus grandis Boheman (Mitlin et al., 1965).

Les affinités biochimiques de l'isotope déterminent très souvent la localisation de celui-ci. Ainsi, l' ${ }^{131}$ Iode semble avoir une affinité spéciale pour la cuticule de l'aphide (KLOFT et al., 1968). Selon le processus de transport, l'élément pourrait d'autre part être assimilé ou catabolisé. C'est pourquoi, compte tenu du type d'expérimentation envisagé, il est indispensable d'étudier au préalable avec précision le choix de la technique, la détermination du niveau d'application, les modalités d'élimination de l'isotope.

GUSS \& BRANSON (1972) utilisent le ${ }^{75}$ Sélénium comme traceur pour estimer les quantités d'une solution comprenant des extraits de Tripsacum floridanum ingérés par Rhopalosiphum maidis Fitch. Ces extraits contiennent en effet une substance qui inhibe l'alimentation du puceron au $1^{\text {er }}$ stade (BRANSON, 1972). Selon la méthode décrite par KIECKHEFER \& DERR (1967), les pucerons s'alimentent dans des enceintes en verre de forme cylindrique (de $2,5 \mathrm{~cm}$ de haut et de $2,5 \mathrm{~cm}$ de diamètre). Les enceintes destinées au contrôle d'excrétion de l'isotope sont fabriquées soit avec du tube clair en celluloïde, soit avec de la matière plastique : dans ce dernier cas, elles sont de construction plus facile et les larves acquièrent à l'intérieur une radioactivité légèrement plus élevée que dans des enceintes en papier. Les cages sont fermées à la base par une double paroi de Parafilm et au sommet par I'habituel sachet à contenu nutritif.

ARN \& CLEERE (1971) emploient la technique du double marquage dans le but de déterminer de manière simultanée les proportions de 2 régimes ingérés par Amphorophora agathonica Hot. Les marqueurs, respectivement le Tritium et le ${ }^{45}$ Calcium, sont introduits par addition aux solutions nutritives de quantités appropriées de ${ }^{45} \mathrm{CaCl}^{2}(20 \mathrm{mc} / \mathrm{mg} \mathrm{Ca})$ ou d'acétate de méthyle tritié $(500 \mathrm{mc} / \mathrm{mmol})$. Un șachet de Parafilm contenant le régime (MITTLER \& DADD, $1964 b$ ) est placé sur l'ouverture d'un capuchon en plastique de $26 \mathrm{~mm}$ de diamètre muni d'un rebord de 
$5 \mathrm{~mm}$. Les 2 régimes alimentaires sont déposés sous forme de gouttes de 5 à $10 \mu \mathrm{l}$ distantes de $1 \mathrm{~cm}$ sur la membrane, de manière à éviter que celles-ci se mélangent. Un $2^{\mathrm{e}}$ capuchon, identique au $1^{\mathrm{er}}$, est placé sous le sachet à contenu nutritif afin de fermer l'enceinte à l'intérieur de laquelle sont disposés les pucerons. Cette méthode pourrait être appliquée à l'étude de 2 substances secondaires.

\section{REMARQUE}

Le Sélénium est toxique envers les vertébrés à une concentration voisine de $5 \mathrm{ppm}$ (MOXON, 1937). Ce radioisotope retarde la croissance de Pseudaletia unipunctata Haworth, à des niveaux diététiques similaires. C'est la raison pour laquelle GUSS \& BRANSON (1972) ont au préalable déterminé les quantités de ${ }^{75} \mathrm{Se} q u i$ n'interfèrent pas au niveau des réponses alimentaires des larves.

Les isotopes peuvent également être utilisés pour des études de comportement sur le végétal. Ainsi EHRHARDT (1967) montre que des feuilles détachées de sycomore absorbent un maximum de ${ }^{32} \mathrm{P}$ au cours d'une période de 6 à 8 h. Mais Periphvllus aceris I. (anciennement Chaetophoria xanthomelas Koch) est lent à s'installer sur des feuilles non infestées. C'est la raison pour laquelle l'auteur est conduit à utiliser le procédé suivant de manière à obtenir un point de départ précis de prélèvement par les aphides $d u{ }^{32} \mathrm{P}$. Dans un $1^{\text {er }}$ temps, les pucerons présents sur une moitié de la feuille sont retirés délicatement du substrat et gardés en vie dans des conditions acceptables d'humidité. Puis le pétiole de la feuille sur laquelle ont été conservés des pucerons est placé dans la solution radioactive pendant $8 \mathrm{~h}$. A l'expiration de ce délai, les aphides qui avaient été au préalable enlevés de la feuille sont déposés de nouveau sur celle-ci où ils s'alimentent rapidement du fait de la présence de leurs congénères, ces derniers étant éliminés $30 \mathrm{mn}$ plus tard. Ce sont par conséquent les individus déposés sur le limbe foliaire à l'expiration du délai de $8 \mathrm{~h}$ qui constituent le point de départ de l'expérimentation. L'emploi de cette méthode d'étude de substances allélochimiques incluses dans la solution avec l'élément traceur (tabl. 2) peut être envisagé pour l'étude du comportement trophique des Homoptères vivant en groupe.

\section{TABLEAU 2}

Les emplois des radioisotopes dans l'étude des aphides (d'après Van EMDEN, 1972).

Some uses of radioisotopes in the study of aphids.

\begin{tabular}{|c|c|c|c|c|}
\hline AUTEURS & NATURE DE L'ÉTUDE & COMPOSÉ RADIOACTIF & APPLICATION & ÉVALUATION \\
\hline CANNY \& ASKham, 1967 & Déplacements des Aphides & ${ }^{14} \mathrm{CO}_{2}-10 \mu \mathrm{Ci}$ & $\begin{array}{l}\text { Par apport continuel dans } \\
\text { une zone de la feuille }\end{array}$ & $\begin{array}{l}\text { Autoradiographie pendant } \\
24 \mathrm{~h}\end{array}$ \\
\hline DANNEEL, 1967 & Sélection de l'alimentation & $\begin{array}{l}{ }^{32} \mathrm{P}-2 \mathrm{mCi} / \mathrm{ml} \\
\text { (composé non énoncé) }\end{array}$ & Régime artificiel & Tube Geiger \\
\hline EHRHARDT, 1967 & Prélèvement alimentaire & ${ }^{32} \mathrm{PO}_{4} \mathrm{NA}_{2} \mathrm{H}-1,4 \mathrm{mCi} / \mathrm{ml}$ & $\begin{array}{l}\text { Tiges coupées et placées } \\
\text { dans la solution contenant } \\
\text { l'isotope }\end{array}$ & Tube Geiger \\
\hline EHRHARDT \& LAMP, 1967 & $\begin{array}{l}\text { Prélèvement alimentaire et } \\
\text { excrétion }\end{array}$ & ${ }^{131} \mathrm{INa}-2,8 \mathrm{mCi} / \mathrm{ml}$ & Régime artificiel & Tube Geiger \\
\hline HENNIG, 1966 & Prélèvement alimentaire & ${ }^{32} \mathrm{PO}_{4} \mathrm{NaH}_{2}-16 \mathrm{mCi} / \mathrm{ml}$ & $\begin{array}{l}\text { Tiges coupées et placées } \\
\text { dans la solution contenant } \\
\text { l'isotope }\end{array}$ & $\begin{array}{l}\text { Tube Geiger et autoradio- } \\
\text { graphie }\end{array}$ \\
\hline KLOFT, 1960 & $\begin{array}{l}\text { Marquage de la salive de } \\
\text { l'aphide }\end{array}$ & $\begin{array}{l}{ }^{32} \mathrm{P} \text { (composé non énoncé) } \\
\mathrm{l} \text { à } 2 \mathrm{mCi} / \mathrm{ml}\end{array}$ & $\begin{array}{l}\text { Tiges coupées et placées } \\
\text { dans la solution contenant } \\
\text { l'isotope }\end{array}$ & $\begin{array}{l}\text { Tube Geiger et autoradio- } \\
\text { graphie }\end{array}$ \\
\hline LAMB et al., 1967 & $\begin{array}{l}\text { Marquage de la salive de } \\
\text { l'aphide }\end{array}$ & ${ }^{86} \mathrm{RbCl}-200-300 \mu \mathrm{Ci} / \mathrm{ml}$ & Régime artificiel & Tube Geiger \\
\hline Moon, 1967 & Prélèvement alimentaire & $\begin{array}{l}{ }^{32} \mathrm{P} \text { (composé non énoncé) } \\
2,5 \mu \mathrm{Ci} / \mathrm{ml}\end{array}$ & Régime artificiel & Tube Geiger \\
\hline STORMS et al., 1967 & $\begin{array}{l}\text { Corrélation entre le prélè- } \\
\text { vement de la radioactivité à } \\
\text { parțir de la plante et la den- } \\
\text { sité de la population des in- } \\
\text { sectes }\end{array}$ & ${ }^{32} \mathrm{PO}_{2} \mathrm{Na}_{2} \mathrm{H}_{2}-5 \mu \mathrm{Ci} / \mathrm{ml}$ & $\begin{array}{l}\text { Solution nutritive incorpo- } \\
\text { porée dans l'eau de culture } \\
\text { d'une plante }\end{array}$ & Tube Geiger \\
\hline
\end{tabular}

Les radioisotopes sont mesurés en Curies $\left(1\right.$ Curie $=3,7 \times 10^{10}$ désintégrations $\left./ \mathrm{s}\right)$ : pour un travail biologique, les millicuries $\left(10^{-3} \mathrm{Curies}\right)$ ou les microcuries $\left(10^{-6}\right.$ Curies) sont normalement utilisées.

Radioisotopes are measured in Curie ( 1 Curie : $3,7 \times 10^{10}$ disintegrations/s) : for biological work, millicuries $\left(10^{-3}\right.$ Curies) or microcuries $\left(10^{-6}\right.$ Curies) are usually used. 


\section{Enregistrement électronique}

MAC LEAN \& KINSEY (1964) ont mis au point un appareil d'enregistrement électronique dans le but d'étudier le comportement alimentaire des Homoptères. Pendant le fonctionnement de l'appareil, l'aphide s'alimentant provoque une variation de résistance consécutive au remplissage des canaux alimentaires et salivaires ménagés dans les stylets maxillaires de l'insecte par de la salive ou de la sève. La source électrique établissant la différence de potentiel est constituée d'un transformateur à filament ; un transformateur secondaire, connecté à un potentiomètre linéaire de 10000 ohms sert à régulariser le voltage. MAC LEAN \& KINSEY font circuler approximativement 20 volts, 60 cycles a.c. dans le substrat. Les changements qui interviennent dans le voltage sont observés sur un oscilloscope et enregistrés sur une bande magnétique. Ce dispositif, modifié par MAC LEAN \& WEIGHT (1968), peut être utilisé pour analyser l'action de substances secondaires incluses dans des solutions nutritives ou circulant dans les tissus du végétal (fig. 8).

En vue de la réalisation d'observations sur la plante, MAC LEAN \& KINSEY (1965) ont utilisé comme matériel une cage rectangulaire de $3 \mathrm{~cm}$ de long, de $1 \mathrm{~cm}$ de large et de $0,8 \mathrm{~cm}$ de profondeur à l'intérieur de laquelle sont enfermés les aphides. Une grille de tungstène doré est collée dans le fond de la cage et la partie inférieure de la grille est recouverte d'une matière isolante. Cette' enceinte est ensuite placée sur le végétal et y est maintenue par l'intermédiaire d'une pince à cheveux. La grille de la cage est connectée à un amplificateur monaural de $25 \mathrm{~W}$; I'oscilloscope à rayon cathodique, la carte d'enregistrement et l'appareil d'audition sont connectés en parallèle au terminal de l'amplificateur de $16 \mathrm{ohms}$; tous les appareils mis en place sont reliés à la terre à l'aide d'un tube de cuivre enfoncé dans le sol. Ce circuit fonctionne à partir du moment où un fil de cuivre étamé est attaché à la partie dorsale du puceron à l'aide d'un conducteur en

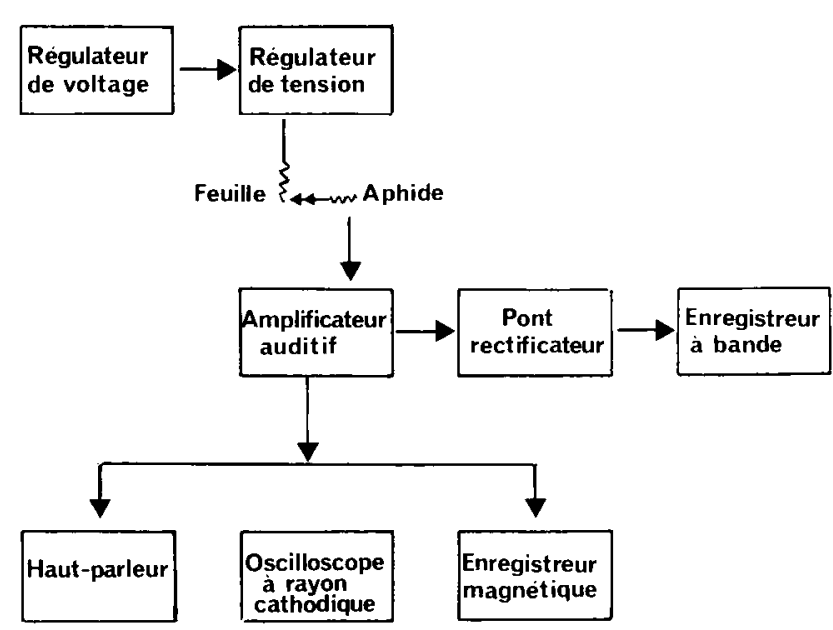

Figure 8

Diagramme de l'appareil servant à déterminer la période de salivation et d'ingestion par l'intermédiaire d'un substrat électriquement conducteur.

Block diagram of apparatus for determining when aphids salivate and ingest within an electrically conductive substrate. argent qui sèche rapidement; ce fil doit avoir une position légèrement incurvée de manière à être en contact avec la grille lorsque l'insecte effectue sa piqûre. Dès l'instant où les canaux des stylets se remplissent de salive ou de liquide provenant du substrat alimentaire, le circuit allant du substrat à l'amplificateur par l'intermédiaire du puceron est fermé.

Sur milieu nutritif, le dispositif mis au point par MAC LEAN \& KINSEY comprend 2 parties : une cage munie d'une grille identique à l'enceinte décrite précédemment et un creuset ou réservoir dans lequel se trouve le liquide nutritif. Ces 2 unités sont construites avec du Plexiglass de 1,6 mm d'épaisseur. Une membrane de Parafilm est étirée sur le fond de la cage séparée de la section contenant le réservoir. Le liquide nutritif est injecté dans le réservoir par l'intermédiaire d'un orifice pratiqué à l'arrière de celui-ci.

MAC LEAN \& KINSEY (1965) ont ainsi réalisé divers graphiques à partir d'une étude effectuée avec A. pisum, lequel a été nourri sur un milieu comprenant des particules d'encre de Chine qui permettent de suivre le transit intestinal du liquide. Salivation et ingestion ont été contrôlées sous la loupe à fort grossissement $(\times 200)$ : les auteurs ont observé que le processus de sécrétion salivaire correspond à une augmentation de voltage et, par voie de conséquence, à une accentuation des courbes sur la bande d'enregistrement. Par contre, la chute du voltage correspond à une ingestion de liquide (fig. 9).

Les légères différences observées sur le végétal (Vicia faba L.) sont dues notamment à la présence d'un nombre moins important de petits pics sur le tracé de la courbe B (fig. 9) ; MAC LEAN \& KINSEY les attribuent à une prise de nourriture plus faible sur le végétal que sur milieu nutritif. Notons à ce sujet la remarque qui a été faite par MITTLER (1958) à la suite de travaux effectués avec Tuberolachnus salignus Gmelin : l'auteur pense que les Aphides ne sucent pas la sève de la plante mais que leur alimentation dépend presque entièrement de la pression exercée par le con-
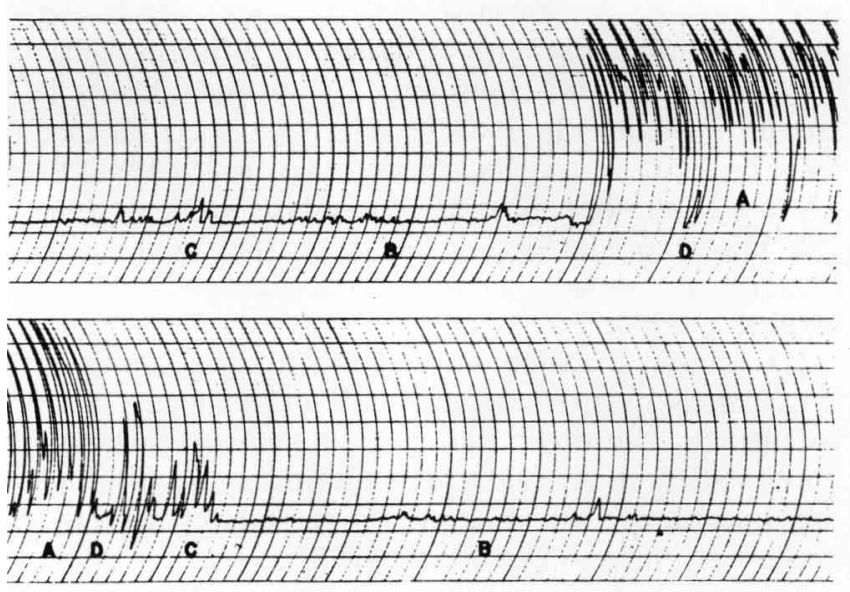

Figure 9

Lecture de droite à gauche d'une bande d'enregistrement concernant le comportement alimentaire d'Acyrtosiphon pisum Harris mis en présence d'une solution à 20 p. 100 de saccharose.

Reading from right to left of a chart recording of the feeding behaviour of Acyrtosiphon pisum Harris probe into $20 \%$ sucrose subs trate. Identification of the curve patterns as follow: A. making of sheath and salivation, $B$. ingestion, $C$. plugging of the food canal, $D$. short ingestion (sampling) (Mc LEAN \& KINSEY, 1965). 
tenu de l'élément criblé, pression qui oblige la sève à monter dans le canal alimentaire de l'homoptère sans que celui-ci ait à faire un effort particulier.

\section{J. Emploi de supports naturels ou artificiels}

Afin d'étudier le comportement alimentaire de l'aphide du fraisier Chaetosiphon fragaefolii Cockerell, SHANKS \& FINNIGAN (1969) utilisent le procédé mis au point par HAGLEY (1967) qui consiste à placer des disques de papier filtre de $13 \mathrm{~mm}$ de diamètre imprégnés de la solution à analyser entre 2 membranes de Parafilm. Seule la membrane supérieure du sachet à contenu nutritif est étirée dans le but d'obtenir un contact aussi étroit que possible avec le papier filtre. Les 2 enveloppes sont ensuite scellées avec un fil métallique chaud. Les mêmes auteurs emploient cette méthode avec des fragments de végétaux.

Etudiant les propriétés phagostimulantes de la sinigrine à l'égard de Brevicoryne brassicae L., WENSLER (1962) fait migrer cette substance dans des feuilles de Vicia faba en trempant celles-ci pendant $24 \mathrm{~h}$ dans une solution aqueuse de sinigrine à $2 \mathrm{p}$. 100. L'auteur utilise également un dispositif comprenant des disques de moelle de sureau imbibés avec une solution aqueuse de sinigrine à 0,2 p. 100 ; ces disques sont ensuite recouverts par une membrane de celloïdine (substance chimique utilisée en microscopie pour couvrir les objets) et sont éclairés par une lumière verte ; celle-ci conduit régulièrement les aphides à effectuer des piqûres d'essai même sur des surfaces qui ne sont habituellement pas éprouvées par l'insecte.

\section{COMPORTEMENT DE L'HOMOPTÈRE SUR DIFFÉRENTS SUBSTRATS}

Le processus de sélection de la plante par un homoptère implique la perception de divers stimuli que KENNEDY \& BOOTH (1951) désignent par les termes de stimuli d'arômes et de stimuli nutritionnels.

Outre les stimuli chimiques, il faut mentionner les stimuli physiques internes et externes visuels ou tactiles : couleur du végétal (CARTIER, 1963 ; MULLER, 1964) ou celle des substrats nutritifs (CARTIER, 19641966) ou encore les aires de hautes résistances aux pénétrations des stylets dans le mésophylle (NAULT \& GYRISCO, 1966).

Les stimuli d'arômes correspondent à des composés sans valeur nutritive propre mais qui engendrent des préférences d'ordre botanique, alors que des stimuli nutritionnels proviennent de substances qui possèdent une importance métabolique de premier plan pour le végétal et l'insecte (KENNEDY, 1958).

MitTLER \& DADD (1963a et 1964b) ont élargi la distinction précédente concernant les stimuli et ont proposé une répartition en 2 groupes : les stimuli gustatifs et les stimuli nutritionnels. Les stimuli gustatifs comprendraient ainsi les stimuli d'arômes (stimuli olfactifs) et des stimuli gustatifs proprement dits tels que les glucosides (KLINGAUF, 1971 ; KUNKEL, 1977 ; MOON, 1967 ; NAULT \& STYER, 1972 ; WenSLER, 1962 ; Van EMDEN, 1973), des alcanes (NöCKER-
WENZEL, 1971) ; des alcaloïdes (SMITH, 1966) et certains composés à valeur nutritive comme le saccharose (MITTLER \& DADD, 1964b), ou certains acides aminés (AuClair J. L. et al., 1957; HARREWIJN P., 1977).

\section{A. Origine de certains stimuli}

Un certain nombre de stimuli (d'arômes externes ou olfactifs) peuvent être détectés grâce aux organes sensibles des antennes (BROMLET \& ANDERSON, 1982). Il est prouvé que l'odeur peut favoriser la découverte d'un hôte (GIBSON \& PLUMB, 1977) et que les périodes de recherches initiales sont influencées par cet hôte (Kennedy et al., 1978 ; Klingauf, 1970 ; Misawa \& HASHIBA, 1967). PetTerson $(1970,1973)$ a constaté que les effluves émises par le benzaldéhyde et la sinigrine en solution aqueuse peuvent être perçues respectivement par des ailés de Rhopalosiphum padi L. et de $B$. brassicae en automne. Des organes sensibles, situés à l'extrémité du labium (ADAMS \& WADE, 1976) des stylets mandibulaires (HARRIS, 1977) de l'organe gustatif épipharyngien interviennent également dans la sélection de l'hôte. Forbes (1977) et TJALLINGII (1976) observent que les microsensilles (mécanorécepteurs) situés à l'extrémité de l'article du rostre interviennent avant qu'il y ait piqûre d'essai, immédiatement après (ou en même temps) qu'il y a reconnaissance de support par le puceron à l'aide de ses tarses. Ceci étant, Wensler (1962), Klingauf (1972), ADAMS \& WADE (1976) pensent que l'acte principal de sélection de la plante intervient après une brève piqûre d'essai.

\section{B. Exemples de critères de sélection de l'hôte par l'aphide}

WENSLER (1962) a observé que $B$. brassicae fait la distinction très nette entre une feuille de féverole, plante qui ne constitue pas une source alimentaire pour l'insecte, et une feuille de rave lorsque les substrats sont protégés par une membrane de celloïdine. A partir de mêmes feuilles placées en position verticale et éclairées sur une seule face, WENSLER constate également que les stimuli émis à partir du substrat sont liés à la réaction du puceron à la lumière.

KLINGAUF (1972) essaie d'établir une relation entre les stimuli reçus durant les visites d' $A$. pisum sur diverses plantes et l'activité locomotrice du puceron. Pour cela, il retient les critères suivants : temps d'errement sur la feuille et durée de la $1^{\text {re }}$ piqûre d'essai, pourcentages d'aphides se trouvant sur la partie supérieure de la feuille et s'orientant vers le côté inférieur au cours du $1^{\text {er }}$ déplacement, attraction exercée par une gamme complète de couleur jaune ou rouge orange.

NAUlT \& STYER (1972), qui ont étudié le comportement de sélection de la plante hôte par les ailés de Lipaphis erysimi Kltb, ont utilisé soit des feuilles de rave ou de féverole, soit des feuilles artificielles confectionnées avec du papier buvard jaune et présentées aux aphides à l'état sec ou humide. L'axe de la feuille fait un angle de $45^{\circ}$ par rapport à la surface du papier support de $27 \mathrm{~cm}^{2}$. La détermination du comporte- 
ment des aphides est basée sur l'observation des déplacements ainsi que sur le nombre et la durée des piqûres d'essai enregistrés pendant $5 \mathrm{mn}$ ou jusqu'à ce que les pucerons s'envolent ou se déplacent hors des limites de la feuille-support.

\section{CONCLUSION}

Cette analyse des principales méthodes d'étude du comportement alimentaire des Homoptères, qui n'a pas l'ambition d'être complète, a pour objet de permettre le meilleur choix de la méthode ou de l'association de procédés d'investigation de la nutrition, problème difficile chez les insectes piqueurs, souvent de petite taille.

Reçu le 25 octobre 1985. Accepté le 19 juin 1986.

\section{REMERCIEMENTS}

Nous exprimons notre reconnaissance à Monsieur DELAPORTE pour la reproduction des figures qui illustrent cette publication.

\section{RÉFÉRENCES BIBLIOGRAPHIQUES}

Adams S. B.. Wade L. W., 1976. Aphid behaviour and host plant preference demonstrated by electronic patterns of probing and fee ding. Am. Potato J., 13, 261-267.

Arn H., Cleere J. S., 1971. A double label choice test for the simul taneous determination of diet preference and ingestion by the aphid Amphorophora agathonica Hotte. Entomol. exp. appl., 14, 377 387.

Auclair J. L., 1965. Feeding and nutrition of the pea aphid Acyrthosiphon pisum Harris (Homoptera, Aphididae) on chemically defined diets of various $\mathrm{pH}$ and nutrient levels. Ann. entomol. Soc Am., 58, 855-875.

Auclair J. L., Cartier J. J., 1963. Pea aphid : rearing on a chemically defined diet. Science $142 ; 1068-1069$.

Auclair J. L., Maltais J. B., Cartier J. J., 1957. Factors in resistance of peas to the pea aphid Acyrthosiphon pisum Harris (Homoptera, Aphididae). II. Amino acids. Can. entomol., 89, 457 464.

Banks C. J., Macaulay E. D. H., 1964. The feeding growth and reproduction of Aphis fabae Scop. on Vicia faba under experimental conditions. Ann. appl. Biol., 53, 229-242.

Branson T. F., 1972. Resistance to the corn leaf aphid in the grass tribe Maydeae. J. econ. entomol., 65, 195-196.

Bromlet A. K., Anderson M., 1982. An electrophysiological study of olfaction in the aphid Nasonovia ribis-nigri. Entomol. exp. appl., 32, 101-110.

Canny M. J., Askham M. J., 1967. Physiological inferences from the evidence of translocated tracer : a caution. Ann. Bot., 31, 409-416.

Cartier J. J., 1963. Varietal resistance of peas to pea aphid biotypes under field and greenhouse conditions. J. econ. Hortic., 56, $205-$ 213.

Cartier J. J., 1966. Aphid responses to colors in artificial rearings. Bull. entomol. Soc. Am., 12, 378-380.

Cartier J. J., Auclair J. L., 1964. Pea aphid behaviour ; colour preference on a chemical diet. Can. entomol., 96, 1240-1243.

Dadd R. H., Krieger D., 1967a. Continuous rearing of aphids of the Aphis fabae complex on sterile synthetic diet. $J$. econ. Entomol., 60, 1512-1514.

Dadd R. H., Krieger D. L., 1967b. Studies on the artificial feeding of the aphid Myzus persicae Sulz. IV. Requirements for water soluble vitamins and ascorbic acid. J. Insect Physiol., 13, 249-272.

Dadd R. H., Mittler T. E., 1965. Studies on the artificial feeding of the aphid Myzus persicae Sulz. III. Some major nutritional requirements. J. Insect Physiol., 11, 717-743.

Dadd R. H., Mittler T. E., 1966. Permanent culture of an aphid on a totally synthetic diet. Experientia, 22, 832 .

Danneel I., 1967. Kurzzeitversuche zur Nahrungswahl von Aphis fabae Scop. (Homoptera, Aphididae). Z. angew. Zool., 54, 181 182 .
Ehrhardt P., 1967. Über die Beziehung der Latenz-larven von Chaetophoria xanthomelas Koch. (Aphididae, Homoptera) zu ihrer Wirtspflanze. Z. angew. Zool., 54, 21-36.

Ehrhardt P., 1968. Die Wirkung verschiedener Spurenelemente auf Wachstum, Reproduktion und Symbionten von Neomyzus circumflexus Buckt. (Aphidae Homoptera Insecta) bei künstlicher Ernährung. Z. verg. Physiol., 58, 47-75.

Ehrhardt P., Lamp K. P., 1967. Versuche zur Aufnahme und Exkretion von J.O.D. 131 bei Aphis fabae Scop. (Aphididae, Homoptera, Insecta). Int. J. appl. Radiat. Isotopes, 18, 543-544.

Forbes A. R., 1977. The mouthparts and feeding mechanism of aphids, 83-103. In Harris K. F. \& Maramorosch K. : "Aphids as virus vectors". Academic Press, New York, 559 p.

Gibson R. W., Plumb R. T., 1977. Breeding plants for resistance to aphid infestation, 473-500. In Harris K. F. \& Maramorosch K. "Aphids as virus vectors". Academic Press, New York, 559 p.

Guss P. L., Branson T. F., 1972. The use of 75 Se in feeding studies with the corn leaf aphid (Homoptera, Aphididae). Ann. entomol. Soc. Am., 65, 303-304.

Hagley E. A. C., 1967. Artificial diet for the adult froghopper Nature, 213, 414-415.

Hamilton M. D., 1935. Further experiments on the artificial feeding of Myzus persicae Sulz. Ann. appl. Biol., 22, 243-258.

Harrewijn P., 1977. Nutritional aspects of development and wing dimorphism in the aphid Myzus persicae. Thesis Inst. V. Plantenziekt. Onderz. Medelel. $N^{\circ} 745$.

Harris K. F., 1977. An ingestion-egestion hypothesis of how circulative virus transmission, 165-220. In Harris K. F. \& Maramorosch $\mathrm{K}$. : "Aphids as virus vectors". Academic Press, New York, $559 \mathrm{p}$

Hennig E., 1966. Zur Histologie und Funktion von Einstichen der schwarzen Bohnenlaus (Aphis fabae Scop.) in Vicia faba. Pflanzen J. Insect Physiol., 12, 65-76.

Hollander J. M., Perlman I., Scabory G. T., 1953. Table of isotopes. Rev. mod. Phys., 25, 469-651.

Honeyborne C. H. B., 1969. An investigation of the responses of aphids to plants treated with growth regulators. $\mathrm{Ph}$. D. Thesis University of Reading.

Kennedy J. S., 1958. Physiological condition of the host plant and susceptibility to aphid attack. Entomol. exp. appl., 1, 50-65.

Kennedy J. S., Booth C. O., 1951. Host alternation in Aphis fabae Scop. Feeding preference and fecundity in relation to age and kind of leaves. Ann. appl. Biol., 38, 25-64.

Kennedy G. G., Mc Lean D. L., Kinsey M. G., 1978. Probing behavior of Aphis gossypii on resistant and susceptible muskmelon. $J$. econ. Entomol., 71, 13-16.

Kieckhefer R. W., Derr R. F., 1967. Rearing three species of aphids on artificial diets. J. econ. Entomol., 60, 663-665.

Kim M., Koh H. S., Ichikawa T., Fukami H., Ishii S., 1975. Antifeedant of barnyard grass against the brown planthopper Nilapar- 
vata lugens Stal. (Homoptera: Delphacidae). Appl. entomol. Zool., 10, 116-122.

Klingauf F., 1970. Zur Wirtswahl der grünen Erbsenlaus, Acyrthosiphon pisum Harris (Homoptera: Aphididae), Z. angew. Entomol., 65, 419-427.

Klingauf F., 1971. Die Wirkung der Glucosids Phlorizin auf das Wirtsvahlverhalten von Rhopalosiphum insertum Walk. und Aphis pomi de Geer. (Homoptera : Aphididae) Z. angew. Entomol., 68, 1, 41-55.

Klingauf F., 1972. Modellversuche zum Einfluss der kurzen Pflanzenbesuche auf das Wirtswahlverhalten während des Befallsfluges von Aphiden. Z. angew. Entomol., 70, 352-358.

Kloft W., 1960. Wechselwirkungen zwischen pflanzensaugenden Insekten und den von ihnen besogenen Pflanzengeweben. Teil. I.Z. angw. Entomol., 45, 337-381.

Kloft V., Ehrhardt P., Kunkel H., 1968. Radioisotopes in investigation of interrelationship between aphids and host-plants, 23-30. In : "Isotopes and radiation in entomology". In Atom. Energy Ag., Proc. Symp. Vienna, 1967, $428 \mathrm{p}$.

Kunkel H., 1977. Membrane feeding systems in aphid research, 311338. In Harris K. F. \& Maramorosch K. : "Aphids as virus vectors'. Academic Press, New York, 559 p.

Kurata F. H., Sogawa S. O., 1976. Sucking-inhibitory action of aromatic amines for the rice plant and leafhoppers. (Homoptera Delphacidae, Deltocephalidae). Appl. entomol. Zool., 2, 89-93.

Lafrance J., 1963. New apparatus and rearing techniques for the study of wireworms (Coleoptera: Elateridae) in organic soils of south western Quebec. Can. Entomol., 93, 1-6.

Lamb K. P., Ehrhardt P., Moericke V., 1967. Labelling of aphid saliva with rubidium 86 . Nature, 214, 602-605.

Mac Lean D. L., Kinsey M. G., 1964. A technique for electronically recording aphid feeding and salivation. Nature, 202, 1358-1359.

Mac Lean D. L., Kinsey M. G., 1965. Identification of electrically recorded curve patterns associated with aphid salivation and ingestion. Nature, 205, 1130-1131.

Mac Lean D. L., Weight W. A., 1968. An electronic measuring system to record aphid salivation and ingestion. Ann. entomol. Soc. Am., 61, 180-185.

Massonie G., 1980. Influence des acides oxaliques et gentisiques sur la valeur alimentaire d'un milieu synthétique. Ann. Nutr. Alim., 34, 139-146.

Misawa T., Hashiba T., 1967. Studies on the mechanism of aphid transmission of stylet-borne viruses. Tohoku J. Agric. Res., 18, 87105.

Mitlin N., Bartlett Alan C., Keller J. C., 1965. Elimination rate and effect on reproduction of ingested radiophosphorus in the boll weevil. J. econ. Entomol., 58, 119-121.

Mittler T. E., 1958. Studies on the feeding and nutrition of Tuberolachnus salignus Gmelin. Homopt. Aphididae. J. exp. Biol., 35, 626-638.

Mittler T. E., 1967. Effect of amino acid and sugar concentration on the food uptake of the aphid Myzus persicae Sulz. Entomol. exp. appl., 10, 39-51.

Mittler T. E., 1970. Uptake rates of plant sap and synthetic diet by the aphid Myzus persicae Sulz. Ann. entomol. Soc. Am. (T.E.) 2, 1701-1705.

Mittler T. E., Dadd R. H., 1962. Artificial feeding and rearing of the aphid Myzus persicae Sulz. on a completely defined synthetic diet. Nature, 195-404.

Mittler T. E., Dadd R. H., 1963a. Studies on the artificial feeding of the aphid Myzus persicae Sulz. I. Relative uptake of water and sucrose solution. J. Insect. Physiol., 9, 623-645.

Mittler T. E., Dadd R. H., 1963b. Studies on the artificial feeding of the aphid Myzus persicae Sulz. II. Relative survival development and larviposition on different diets. J. Insect Physiol., 9, 741-757.

Mittler T. E., Dadd R. H., 1964a. An improved method for feeding aphids on artificial diets. Ann. entomol. Soc. Am., 57, 139-140.

Mittler T. E., Dadd R. H. 1964b. Gustatory discrimination between liquids by the aphid Myzus persicae Sulz. Entomol. exp. appl., 7, 315-328.
Mittler T. E., Dadd R. H.. 1964c. Assimilation by apterous adult Myzus persicae maintained on a synthetic diet. Ann. entomol. Soc. Am., 58, 396-401.

Mittler T. E., Dadd R. H., 1965. Difference in the probing responses of Myzus persicae Sulz. elicited by different feeding solution behind a parafilm membrane. Entomol. exp. appl., 8, 107-122.

Montgomery M. E., Arn H., 1974. Feeding response of Aphis pomi, Myzus persicae and Amphorophora agathonica to phlorizin. J. Insect Physiol., 20, 413-421.

Moon M. S., 1967. Phagostimulation of a monophagous aphid. Oikos, 18, 96-101.

Moxon A. L., 1937. Alkali disease or selenium poisonning. South Dak. agr. exp. Stn. Bull., 311-391.

Muller H. J., 1964, Über die Anfluchtdichte von Aphiden auf farbige Salatplanzen. Entomol. exp. appl., 7, 85-104.

Nault L. R., Gyrisco G. G., 1966. Relation of the feeding process of the pea aphid to the inoculation of pea enation mosaic. $A n n$. entomol. Soc. Am., 59, 1185-1197.

Nault L. R., Styer W. E., 1972. Effects of sinigrin on host selection by aphids. Entomol. exp. appl., 15, 423-437.

Nickel J. I., Sylvester E. S., 1959. Influence of feeding time, stylet penetration and developmental instar on the toxic effect of the spotted alfalfa aphid. J. econ. Entomol., 52, 249-254.

Nöcker-Wenzel K., 1971. Isolierung von Oberflachensubstanzen aus Vicia faba L. in Rahmen von Untersuchungen zur InsektWirtspflanzen Beziehung. Tetrahedron letter, 46, 4409-4412.

Pettersson J., 1970. Studies on Rhopalosiphum padi. Lantbrukshöyisk Annlr., 36, 381-399.

Pettersson J., 1973a. Olfactory reactions of Brevicoryne brassicae L. Swed. J. agric. Res., 3, 95-103.

Pettersson J., 1973b. Aphids and host plant communication. Symb. bot. Uppsala, 22, 107-113.

Sekido S., Sogawa K., 1976. Effects of salicylic acid on probing and oviposition of the rice plant and leafhoppers (Homoptera Delphacidae and Deltocephalidae ). Appl. entomol. Zool., 11 (2), 7581 .

Shanks C., Finnigan F., 1969. Behavior, survival and reproduction of the strawberry aphid and holidic diets and nonhost plants. Ann. entomol. Soc. Am., 62, 6, 1490-1492.

Smith B. D., 1966. Effects of the plant alkaloid sparteine on the distribution of the aphid Acyrthosiphon spartii Koch. Nature, 212, 213-214.

Sogawa K., Patkak M. D., 1970. Mechanism of brown planthopper resistance in Mutgo variety of rice (Homoptera, Delphacidae). Appl. entomol. Zool., 5, 145-158.

Storms J. J. H., Harrewijn P., Noordink J. Ph. W., 1967. A new approach to the physiological host plant parasite relationship. A technique in the field of applied entomology. Neth. J. Plant Pathol., 73, 165-169.

Tjallingii W. F., 1976. Mechanoreceptors for the aphid labium. Mededelingen Fac. Landbouww. Rijksuniv. Gent, 41 (2, 1 ; 2, II) ; $(12+), 465-494(5+), 995-1542$.

Van Emden H. F., 1967. An increase in the longevity of adult Aphis fabae fed artificially through parafilm membrane on liquids under pressure. Entomol. exp. appl., 10, 166-170.

Van Emden H. F., 1972. Aphid technology. Acad. Press, London and New York, $334 \mathrm{p}$.

Van Emden H. F., 1973. Aphid host plant relationship. 54-75. In A. D. Lowe: "Perspectives aphid biology". N. 2 Entomol. Soc. Bull., 2, 123 p.

Wearing C. H., Van Emden H. F., 1967. Studies on the relations of insect and host plant. Effects of water stress in host plants on infestation by Aphis fabae Scop. Myzus persicae Sulz. and Brevicoryne brassicae L., Nature, 213, 1051-1052.

Wensler R. J. D., 1962. Mode of host selection by an aphid. Nature, 195, 830-831.

Yoshihara T., Sogawa K., Patkak M. D., Juliano B. O., Sakamura S., 1979. Soluble silicic acid against the planthopper Nilaparvata lugens Stål (Homoptera, Delphacidae). Entomol. exp. appl., 26 (3), 314-322. 